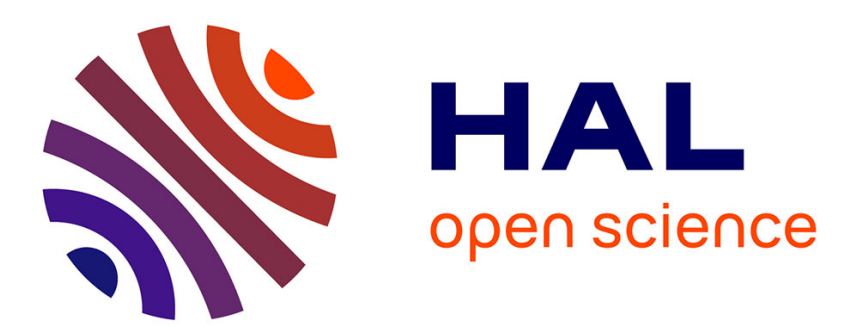

\title{
Les interventions précoces de développement chez les nouveau-nés prématurés : une prise en compte perfectible
}

\author{
Jeremy Rollin
}

\section{- To cite this version:}

Jeremy Rollin. Les interventions précoces de développement chez les nouveau-nés prématurés: une prise en compte perfectible. Kinésithérapie, la Revue, 2020, 20 (228), pp.3-10. 10.1016/j.kine.2020.06.015 . hal-03205446

\section{HAL Id: hal-03205446 \\ https://hal.science/hal-03205446}

Submitted on 22 Apr 2021

HAL is a multi-disciplinary open access archive for the deposit and dissemination of scientific research documents, whether they are published or not. The documents may come from teaching and research institutions in France or abroad, or from public or private research centers.
L'archive ouverte pluridisciplinaire HAL, est destinée au dépôt et à la diffusion de documents scientifiques de niveau recherche, publiés ou non, émanant des établissements d'enseignement et de recherche français ou étrangers, des laboratoires publics ou privés. 


\section{Les interventions précoces de développement chez les nouveau-nés prématurés : une prise en compte perfectible}

Jérémy Rollin

\section{Introduction}

Près de 800000 bébés naissent par an en France, dont la quasi-totalité en milieu hospitalier [1]. Un bébé naît physiologiquement entre 37 et 41 semaines d'aménorrhée (SA) mais 50000 enfants par an naissent prématurément selon l'INSERM[2], dont $10 \%$ de grands prématurés (de 28 à $32 \mathrm{SA}$ ) et $5 \%$ de très grands prématurés (en-dessous de 28SA). La prématurité entraîne des risques immédiats pour la survie (risques cardiaques, respiratoires et digestifs) augmentant avec des faibles poids et des plus jeunes âges de naissance. Ainsi, les nouveau-nés prématurés jugés à risque restent à l'hôpital sur une longue durée, parfois jusqu'au terme théorique, dans un contexte de médicalisation lourde, avec d'éventuels problèmes de séparation parents-enfant au profit des machines nécessaires à la survie [3-5] (couveuse, parfois avec assistance respiratoire, alimentation par sonde naso-gastrique et monitoring cardio-respiratoire).

Les problèmes immédiats ne sont pas les seuls enjeux. En France, le long recul temporel sur l'étude Epipage 1 montre des différences entre prématurés et enfants nés à terme avec un moindre Quotient Intellectuel (QI), des troubles de l'attention et des apprentissages et des difficultés motrices à l’âge scolaire [6].

Pour lutter contre l'apparition de ces troubles, plusieurs interventions précoces de développement [7] peuvent être proposées : en milieu hospitalier, souvent sous le vocable «soins de développement»[8] et pratiqués de manière pluridisciplinaire, on retrouve l'optimisation de l'échange et de la communication (tactile, orale...) avec le bébé [5,9], le kangourou care [10] ou le « peau à peau» [11] direct ou via une écharpe [12], le développement de l'oralité $[9,13,14]$, des massages réguliers $[15,16]$, le travail précoce de regroupement vers la flexion [9,17], les changements de positions réguliers ou la formalisation de protocoles NIDCAP dans les services [18,19]. Ces soins peuvent se poursuivre en ville, de manière privilégiée par les masseurs kinésithérapeutes (MK) [7], et intégrer progressivement des notions plus actives ou posturales quant aux propositions d'enroulement, puis de guidage de la motricité $[9,13]$. Mais cette séparation hôpital-ville est 
plus de l'ordre de l'organisationnel et le vocable interventions précoces de développement recoupe l'ensemble de ces actions [20].

En tant que praticien libéral, de manière empirique, je voyais les enfants arriver des semaines, parfois des mois, après leur sortie d'hôpital. Même si ces délais peuvent s'expliquer par l'incertitude médicale et l'attente de signes cliniques plus évidents, le peu de connaissances des parents quant aux soins de développement m'ont interrogé alors qu'il existe des aides aux diagnostics précoces comme l'analyse des mouvements généraux [21]. Or, les périodes sensibles de Dehaene [22] mettent en évidence des fenêtres de plasticité cérébrale en dehors desquelles les modifications deviennent plus difficiles. Le bon respect de la temporalité des prises en charge apparait donc comme un enjeu dans ces soins [7], faisant l'objet de recommandations de la Haute Autorité de Santé [23]. Ainsi la recherche des causes de ces lenteurs de prises en charge a été l'objet de ce travail. 


\section{Population et méthodes}

Cette recherche est un travail sociologique. L'enjeu n'était pas de quantifier le phénomène, mais de l'expliquer par son analyse en milieu réel. Dans ce cadre, entretiens [24,25] et observations [26] sont des outils permettant d'approcher finement la réalité à objectiver.

La principale particularité de cette méthode de recherche est l'immersion du chercheur dans le milieu exploré. Il ne peut alors que donner sa vision personnelle de la situation, à travers le prisme de son expérience du réel et de l'axe d'observation choisi [27]. Même si une réflexivité est de mise [28], concourant à «mettre à jour ses prénotions » et « traiter les objets sociaux comme des choses » [29], il n'en reste pas moins que l'identification du positionnement du chercheur est nécessaire à l'analyse faute d'une distanciation totale de l'enquêteur envers l'enquêté [30] (surtout que les données issues de ce travail, réalisé dans un cadre académique, étaient analysées par le seul auteur). Ainsi, je suis un homme, masseurkinésithérapeute (MK) pédiatrique libéral intégré de longue date dans le réseau de soins périnataux régional. Cette connaissance du milieu m'a permis un accès facile aux structures de soins enquêtées, aux professionnels de santé et aux parents. C'est cet «opportunisme méthodologique » [31] qui a présidé au choix géographique (ville de province, avec un CHU, mais un nombre de cabinets de rééducation pédiatrique restreint, puis élargissement à un autre CHU de la même région). Il a, par contre, l'inconvénient de masquer certaines réalités quotidiennes, trop souvent observées [29,32].

Dans une démarche inductive, le premier terrain a été l'arrivée des familles en cabinet de rééducation de ville. Ce terrain, trop connu, était peu favorable à des observations ethnographiques distanciées [33]. Aussi, il a été préféré la méthode d'entretiens ethnographiques (au sens de Béaud [24], c'est-à-dire reprenant à la fois les éléments biographiques concourant à l'explication du phénomène à élucider et les éléments contextuels -positionnement vis-à-vis de la rééducation, vis-à-vis du vécu hospitalier...-) longs et très libres pour ne pas imposer de problématiques présupposées aux enquêtés [34].

Les critères d'échantillonnage, dans une approche exploratoire ont été très souples :

-choix genré (choix des mères, au vu de la répartition des tâches familiales encore la plus fréquente dans notre organisation sociale $[35,36])$

-choix de se limiter à des mères de bébés prématurés ayant une indication de rééducation, pour étudier ainsi les suivis d'enfants jugés les plus à risque, même si cela exclu de fait des familles qui auraient refusées ce type de soin. Matériellement, ce choix a entrainé le recrutement des mères enquêtées au sein des deux cabinets de rééducation pédiatrique de la 
ville (listés par le réseau de périnatalité régional, ils ont été pensés comme intermédiaires efficaces pour rencontrer ces populations dans un temps contraint)

-sans nombre préétabli, laissant libre cours à l'apparition de l'effet de saturation autour de certains points redondants [25].

-les entretiens se sont déroulés au sein des cabinets, dans une pièce isolée, avec une présentation du chercheur comme MK s'intéressant aux circonstances périnatales dans un cadre académique (1 seule mère était connue -une séance de kinésithérapie réalisée ensemble, préalablement à l'entretien-, les autres ont été recrutées via demandes orales des MK des deux cabinets, après présentation du projet de recherche)

Les 8 premières mères s'étant présentées en novembre et décembre 2017 ont toutes décrit la même trajectoire hospitalière, dont 6 à travers des entretiens très détaillés [24], d'une heure à une heure et demi (les 2 autres refusant, de par la difficulté à réévoquer en détail et sous enregistrement ces épisodes, décrits comme «douloureux»). Tous les entretiens se sont déroulés en une seule fois avec enregistrement sonore et retranscription ultérieure (sans relecture par les enquêtées), quelques prises de notes contextuelles les complétant (positionnement générale de l'enquêtée, difficulté à répondre aux questions ou participation enthousiaste...). Si la thématique était leur accouchement et ses suites, le fil par lequel le raconter était à leur initiative. En dehors de relances, la seule intervention était de ramener la discussion à leur cas précis quand il y avait des propos d'ordre généraux et de faire évoluer chronologiquement le récit. Il n'y a pas eu de codages des verbatims mais relectures par le chercheur et dégagement des redondances dans les parcours sur les seules thématiques liées aux interventions précoces de développement (présence de ces soins à l'hôpital, moment de leur instauration le cas échéant, liaisons soins hospitaliers-soins de ville sur ces thématiques, mise en place de ces soins en ville). Devant l'uniformité des positionnements, il n'a pas semblé pertinent d'en réaliser plus [37] dans les contraintes de temps de cette recherche, mais plutôt de tenter de comparer ces données à celles produites par un travail d'observation ethnographique.

Les observations ethnographiques de ce second terrain ont été réalisées en néonatalogie et dans les consultations médicales hospitalières de suivi: une dizaine de sessions d'observations ont été réalisées (demi-journées ou journées), à différentes périodes de la semaine, sur une durée de 2 mois en janvier et février 2018 (Deux CHU A et B distincts ont été concernés pour comparer les comportements dans deux structures distinctes et ne pas conclure sur des phénomènes qui auraient pu être dus à une organisation singulière, il n'y a 
pas eu de refus de participation) [38]. L'accord des chefs de service a été obtenu (échange par mail) suite à une présentation de la recherche. Bien que cette présence soit strictement circonscrite au cadre d'une recherche en sciences sociales, la possession du diplôme de MK leur était connue. Cette présentation de la recherche et des diplômes de l'enquêteur a aussi été fait aux personnels et aux parents présents durant les observations. Des notes ont été prises «à la volée » durant ces périodes et seront retranscrites ici sans modification, afin de ne pas les travestir et de conserver le ton et les termes considérés comme adéquat sur le moment en fonction du contexte vécu, dans une démarche strictement anthropologique [39] (sur support carnet-papier en service, tapé sur ordinateur ultérieurement, directement sur ordinateur durant les consultations en bureau, sans relecture de la part des enquêtés). Les éventuels ajouts ou précisions indispensables à la compréhension ont été mis entre crochets. L'attention a été portée sur les situations en lien avec les interventions précoces de développement (réalisation par les familles, les soignants, discussions à ce propos entre soignants), les discussions ou démonstrations mettant en jeu parents et soignants, ainsi que sur les prescriptions médicales. C'est leur présence ou leur absence qui ont été rapportées à chaque observations, avec les explications visibles de ces états de fait. Souvent des discussions en service ont donné lieu à des entretiens informels avec d'autres mères et avec des personnels soignants de diverses professions, avec prises de notes manuelles [40]. En complément, 4 entretiens formels, enregistrés, ont été réalisés avec des soignants (2 puéricultrices, 1 interne et $1 \mathrm{MK}$ ), dans des endroits isolés des services. Ces personnels ont été choisis parce que les observations semblaient montrer un intérêt appuyé pour les soins de développement. Les propos ont soutenu l'analyse mais ne sont pas présentés ici. En effet, pour objectiver l'activité quotidienne, l'observation directe a plus force de preuve que la projection que pourrait en faire l'enquêté au cours d'un entretien [30]. L'analyse interprétative a été faite par comparaison des observations directes et de la littérature des sciences sociales en santé, pour tenter de dégager des phénomènes sociologiques à travers les situations particulières perçues.

Le faible nombre d'enquêtés (mères, mais aussi soignants dans les observations ethnographiques) présente l'avantage d'explorer avec précision leurs parcours, actions et représentations. C'est ce travail de terrain minutieux qui permet de proposer des réponses à la problématique initiale par la mise en perspective de points de vue d'acteurs différents, mais travaillant sur les mêmes situations (ici les soins aux bébés). Cette méthodologie est donc partiellement anti-positiviste, cherchant à récolter une réponse sur le terrain plutôt qu'à élaborer une théorie «hors sol » puis de la tester. L'écueil évident étant, à l'inverse, un excès 
d'intuitionnisme [30], c'est-à-dire le risque de généralisation hâtive... Mais la comparaison avec la littérature sur les sujets d'études proches permet de limiter partiellement ce risque. Dans tous les cas, la méthode sociologique qualitative et inductive ne peut nier l'impossibilité de saisir l'intégralité du sujet, ce qui oblige à utiliser le singulier pour éclairer la généralité [41]. 


\section{Résultats}

Les entretiens avec les mères comme indices d'un manque d'informations sur les soins de rééducation précoce

Les enquêtées comprenaient des primipares (4) et des multipares (2), des mères de jumeaux (1) ou de bébé unique (5). Les naissances se sont toutes produites entre $28 \mathrm{SA}$ et $34 \mathrm{SA}$, avec un minimum de 3 semaines d'hospitalisation pour les nouveau-nés.

Les professions des parents ont été pris comme indicateurs de niveau social (Tableau 1).

Tableau 1 : professions des parents des bébés dont la mère a accepté un entretien formel.

\begin{tabular}{|l|l|l|l|l|l|l|}
\hline Bébés & $\begin{array}{l}\text { Adam et } \\
\text { Bernie }\end{array}$ & Adèle & Béatrice & Caroline & David & Esteban \\
\hline $\begin{array}{l}\text { Profession } \\
\text { de la mère }\end{array}$ & $\begin{array}{l}\text { Conseillère } \\
\text { en } \\
\text { économie } \\
\text { sociale et } \\
\text { familiale }\end{array}$ & $\begin{array}{l}\text { Employée } \\
\text { de } \\
\text { restauration }\end{array}$ & $\begin{array}{l}\text { Mère au } \\
\text { foyer } \\
\text { (études } \\
\text { d'infirmière) }\end{array}$ & Infirmière & Enseignante & $\begin{array}{l}\text { Employée } \\
\text { de }\end{array}$ \\
restauration \\
$\begin{array}{l}\text { Profession } \\
\text { du père }\end{array}$ & Artisan & $\begin{array}{l}\text { Artisan (en } \\
\text { invalidité) }\end{array}$ & Commercial & Médecin & Enseignant & $\begin{array}{l}\text { Aide- } \\
\text { soignant }\end{array}$ \\
\hline
\end{tabular}

Leur hétérogénéité (signant des patrimoines culturels et économiques variés [42] et des niveaux d'interconnaissances et de mutuelles compréhensions différentes avec les soignants ou le chercheur [43-45]) est un élément de poids crédibilisant les vécus homogènes quant aux soins proposés durant l'hospitalisation.

L'ouverture permise par des entretiens semi directifs approfondis a permis de détecter chez toutes les mères un discours commun autour du manque d'information sur les interventions précoces de développement (Extraits 1, 2 et 3 à titre d'exemples).

Ils permettent de relever un manque d'empowerment des parents [46] et de soutien à leur implication dans ces soins à l'hôpital et en vue du retour à domicile.

Pour autant, ces entretiens sont insuffisants à eux seuls pour conclure à l'absence de présentation des interventions précoces de développement à l'hôpital, car ne décrivant pas les 
situations réelles vécues, mais simplement la construction rétrospective qu'en ont fait les parents $[30,33]$

"Enquêteur : Est-ce que pendant ce premier mois vous avez eu des conseils? Des conseils de stimulation, des conseils d'alimentation, des conseils de sommeil ?

Maman : Pas du tout. Quand je suis rentrée à la maison vous voulez dire?

Enquêteur : Oui

Maman : Pas du tout. Même quand on est sorti, au niveau de l'alimentation, ils nous ont dit vous prenez le lait que vous voulez. »

Extrait 1 : entretien avec une maman de jumeaux (Adam et Bernie)

«Enquêteur : Et du coup quand vous étiez à l'hôpital l'après-midi et qu'elle dormait vous... Qu'est-ce que vous faisiez?

Maman : Ben comme on l'avait sur nous, voilà, on attendait que ça passe, que le temps passe...

Enquêteur : Elles vous avaient pas donné de conseils de jeux ou de portage ou de...

Maman : Non.

Extrait 2 : entretien avec une maman d'une petite fille (Adèle)

«Avant de sortir on nous avait dit comme conseils genre: installer le bébé sur le dos quand il dort, on a eu droit à " pas de tours de lits », «la chambre à $20^{\circ} \mathrm{C} »$, enfin bref, j'en passe mais pas de conseils en termes de stimulations, euh d'apprentissages moteurs, psychomoteurs, tout ça, rien du tout. Ça c'est débrouillez-vous comme vous pouvez, voyez avec votre pédiatre [petit sifflement] On éjecte. »

Extrait 3 : entretien avec une maman d'une petite fille (Béatrice)

$\underline{\text { Les observations comme confirmation de la faible part des soins de développement et de leur }}$ promotion en milieu hospitalier

L'hôpital joue un rôle aujourd'hui réorienté vers le traitement du soin aigu, complexe, immédiat [47,48]. Ce qui se traduit, concernant les nouveau-nés prématurés, par la priorisation des soins en liens avec la survie. Il convient de rappeler que ce rôle est tenu avec une grande efficacité, en témoigne les taux de survie importants relevés par les études Epipage [6,49], même pour les très grands prématurés [50]. Ainsi la nature même de la prise 
en charge néonatalogiste des nouveau-nés prématurés concoure à hiérarchiser les soins, au profit du maintien des fonctions digestives (reflux gastro-œsophagiens, vomissements), respiratoires (oxygénothérapie rendant les mobilisations difficiles) et cardiaques (fatigabilité d'un point de vue général ou limitation des mouvements possibles avec les oxymètres de pouls)

Concernant les soins de développement à l'hôpital et leur continuité en ville sous la forme de séances de kinésithérapie précoces, le constat est plus mitigé. Lors des entretiens (formels ou informels) une partie des médecins interrogés ont l'impression de bien prescrire ou informer les familles sur ces soins (pour notre terrain, les plus âgés et expérimentés). Les observations, elles, ont tendance à relativiser leurs perceptions : ils se retrouvent parfois à gérer des problèmes logistiques, médicaux ou alimentaires plus immédiats, reléguant les soins de développement à un « après ».

En fin de journée, j'assiste aux transmissions entre pédiatres :

Ils évoquent les cas, bébé par bébé. Les informations portent sur ce qui s'est passé dans la journée, notamment les problèmes qui se sont posés et les examens médicaux prévus ou reçus. Mais aussi les demandes ou sujets d'inquiétude avec les parents.

« maman inquiète, elle dit qu'on ne fait pas téter sa fille [et qu'elle est nourrie par voie naso gastrique] J'ai pas trop envie que la maman ne soit pas trop contente, donc j'espère qu'elles [les puéricultrices] vont la stimuler un peu. »

Ils évoquent aussi les permissions de sortie, le besoin d'une feuille avec horaires des médicaments pour une maman, les retours d'une autre "Ça se passe bien à la maison ».

$[\ldots]$

Ensuite les discussions concernent la rédaction des prescriptions en avance pour les enfants stables. "Pour soulager le travail du week end. »

Une seule allusion (succincte!) à la situation motrice pour un bébé :

«Il restait avec une hypotonie axiale... Toute façon lui faut l'inclure dans le réseau, tout ça... »

Extrait 4 : notes lors d'une observation en service de neonatalogie, CHU A

De plus, en dehors de l'importance première du rôle médical strict de normalisation des paramètres digestifs, cardiaques et respiratoires, les soins de développement peuvent être une notion un peu vague pour certains médecins et infirmiers de ces services. En effet, malgré une 
croyance commune dans les connaissances reconnues par un titre académique [51-53], la réalité objective rappelle que les hétérogénéités de savoirs sont réelles, à fortiori quand on s'éloigne des cœurs de métiers.

Ensuite la discussion s'engage avec une pédiatre et une interne sur la question de qui a appelé le kiné pour le petit dont j'ai suivi la séance. Il s'agit du médecin rééducateur qui passe de temps en temps dans le service. Devant mes questions, et n'en sachant pas beaucoup plus, elles me montrent le mot laissé lors de son passage, demandant des soins kiné pour une « hypotonie axiale » [il n'y a pas de bilan accessible dans le service].

Je discute avec elles de leurs critères d'appels. Elles me disent ne pas être trop au point sur le sujet et laisser plutôt le médecin rééducateur faire [Un seul médecin rééducateur pédiatrique pour tout le CHU]. En développant, elles me disent que souvent il s'agit de grande prématurité, donc de petits qui ont été suivis d'abord en réa néo nat où la kiné a été mise en place.

J'essaie de savoir ce qui pourrait les alerter à demander des soins de développement précoces mais elles disent être trop nouvelles pour avoir assez d'expérience dans le domaine. Par contre elles citent les problèmes médicaux «étiquetés» (plagiocéphalie...) comme demandant alors des « soins kiné ».

Nous discutons ensuite soins de développement sur lesquels elles ne se sentent pas très compétentes («nous on fait plutôt le médical »), mais l'interne précise qu'au CHU B [nom des hôpitaux modifiés] «ils sont très à cheval là-dessus avec le protocole Nidcap ».

Suite à ma question, l'interne ne connaît pas les travaux sur l'analyse des mouvements généraux [examen précoce des troubles de développement].

Elles me disent alors regretter l'absence de kiné ou psychomot pour s'occuper de ces choses-là qu'elles ne peuvent gérer [Une MK intervient sur prescription mais n'est pas présente en permanence].

Extrait 5 : notes lors d'observation en service de néonatalogie, CHU A

Revenu en "salle de contrôle " j'interroge les puéricultrices à ce propos [le peau à peau] : elles me disent que les bras ou le peau à peau c'est des sorties de couveuse stressantes donc à limiter et à faire pour une durée d'au moins une heure pour que le stress que ça engendre soit compensé par les bienfaits «psy».

On discute ensuite des soins de développement et elles reconnaissent leur nécessité avant « maturité ». Par contre, pour elles, quand ils sont " à terme », plus besoin : " c'est comme les 
bébés à terme ».

$[\ldots]$

Je les interroge sur les retournements [changements de postures], si il s'agit de soins de développement. Elles conviennent qu'il faut le faire y compris en néo nat « si il y a le temps ».

Extrait 6 : notes lors d'observation en service de néonatalogie, CHU A

A travers ces exemples, cherchant à identifier le contenu concret des actions en matière d'interventions précoces de développement, on s'aperçoit que le répertoire d'actions est bien plus faible ou aléatoire que l'arsenal thérapeutique théorique disponible, et que le bilan précoce lui-même peut être assez flou.

Ce schéma se retrouve aussi bien en service qu'en consultation de suivi hospitalier, où le protocole médical, tel qu'il a pu être observé, (auscultation, mesurage, pesée, vision, tests codifiés, remplissage du dossier) ne laisse que peu de place à l'évaluation directe du savoir des parents en termes de soins de développement. Ceux-ci réagissant, dans l'interaction, aux préoccupations du médecin, mais ne l'entraînant que rarement sur des sujets non abordés.

La concordance des témoignages des mères et des observations réalisées sur la prépondérance de l'action médico centrée par rapport aux interventions précoces de développement in situ et post hospitalisation démontre la réalité du partage des tâches hospitalières [54]. La place des MK dans ces services ne leur permet pas d'imposer les soins de développement comme problématique incontournable, malgré l'obligation de leur présence en service [55].

En effet, ceux-ci n'ayant pas d'accès direct aux nouveau-nés prématurés, ils doivent recevoir une prescription médicale pour intervenir. Quant aux autres spécialistes de la rééducation, ils sont soit trop peu nombreux (médecins rééducateurs) soit moins reconnus par le législateur et n'ayant pas non plus d'accès direct (psychomotriciens par exemple), ne pouvant ainsi pas intervenir plus systématiquement et librement.

Ainsi le maintien d'une hiérarchisation des professions (objectivable à travers le lien de prescription) conjugué à l'organisation hospitalière laissant peu de temps aux médecins pour dépasser les enjeux vitaux immédiats [56] rend, de fait, plus difficile la prise en compte d'autres aspects du développement de l'enfant. 
Ces aspects finissent par être pris en compte (les mères rencontrées avaient leurs bébés pris en charge), mais leur faible mise en avant dans les premiers temps de la vie est défavorable à un investissement rapide des familles dans ce type de soin, une fois rentrées à domicile. 


\section{Discussion}

La force de ces résultats est leur concordance avec les travaux mettant en évidence le poids des politiques managériales hospitalières (hiérarchisant les soins) [57-59] et celui de la domination médicale sur les autres personnels de santé (rendant difficile l'imposition de logiques de soins différentielles sans leur accord, symbolisé, d'un point de vue légal, par l'ordonnance)[60-63]. Ceci explique la difficulté des MK à imposer les interventions précoces de développement comme problématique prioritaire.

L'autre concordance est interne à l'enquête : l'effet de saturation constaté dans les entretiens avec les mères, unanimes quant au manque d'information sur les soins de développement, recoupe des observations similaires dans deux CHU très différents (en termes de personnels, de logiques hiérarchiques ou d'investissements dans le NIDCAP). Ces concordances avec des outils, des lieux, des enquêtés différents argumentent l'unicité d'un fait: un manque de sensibilisation des parents aux soins de développement mais ne tranchent pas sur les causes de ce manque.

La réflexivité du chercheur sur l'organisation sociale de la santé a été un outil pour limiter l'impact d'opinions personnelles sur l'objet de recherche mais il serait illusoire d'imaginer pouvoir s'extraire totalement d'un monde social dans lequel nous évoluons. L'intérêt de cette immersion du chercheur dans le milieu est sa capacité à moins le modifier par sa présence que ne pourrait le faire un anthropologue extérieur et donc à le saisir dans son organisation écologique habituelle [64]. Le revers de la médaille est que mon parcours sociologique et mon identité au sein d'une profession en quête d'émancipation ont pu favorisé la mise en évidence privilégiée des logiques de domination, qu'elles soient interprofessionnelles (domination médicale) ou managériales (protocolisations, cotations, voire dotations privilégiant les actes vitaux, les personnels de soins aigus, manque de temps de formation continue $[65,66])$. Si celles-ci sont indéniables, elles mériteraient pourtant un approfondissement : la littérature rappelant que des causes aussi diverses qu'un paternalisme médical $[67,68]$, un manque de personnel dû à des budgets comprimés [7,56,58], un manque de connaissance sur les compétences professionnelles de l'autre [69] ou un décalage entre action pensée et action effectuée sont autant de logiques très différentes [70] compliquent certains soins, souvent les moins quantifiables [59]. 
Enfin, se pose, comme dans tout travail scientifique, la question de la généralisation d'observations locales. Celle-ci n'est possible avec robustesse qu'en associant étroitement analyse qualitative et travail quantitatif sur plus de structures, ce qui n'a pas été fait ici. 


\section{Conclusion}

Le soin n'est pas simplement la proposition formelle de traitements objectivés par la recherche clinique. Il implique aussi la prise en compte du contexte culturel, social, organisationnel, à la fois familial et des personnels soignants.

Ce contexte est à l'origine d'allongement des délais de prise en charge des nouveau-nés prématurés. Ce travail souligne une origine systémique, par une priorisation des soins aigus hospitaliers empêchant la mise en avant optimale des interventions précoces de développement.

Pour améliorer la situation et concilier les impératifs immédiats et de longs termes, la place du MK doit sans doute être repensée, afin que son regard clinique se surajoute à celui du médecin, pour dédoubler ou élargir le diagnostic. Se posera alors la question de qui décide du statut d'enfant normal ou pathologique [71,72]. 


\section{Références}

[1] Jacques B. Sociologie de l'accouchement. 1ère édition. Paris: Presses universitaires de France; 2007.

[2] Ancel P-Y, Rozé JC. Prématurités, ces bébés qui arrivent trop tôt 2015.

[3] Mathelin C. Parents et soignants autour du bébé prématuré. Émotions Autour Bébé, ERES; 2006, p. 143-148.

[4] Feldman R, Olds SW, Papalia D. Psychologie du développement humain. Bruxelles: De Boeck; 2010.

[5] Dugnat M. Troubles relationnels père-mère-bébé quels soins? S.1.: Arip : Eres; 2001.

[6] Larroque B, Delobel M, Arnaud C, Marchand L. Devenir à 5 et 8 ans des enfants grands prématurés dans l'étude Épipage: développement cognitif, troubles du comportement et scolarisation: Outcome at 5 and 8 years of children born very preterm. Arch Pédiatrie 2008;15:589-591.

[7] Mellier D, Marret S. Soigner et prendre soin des enfants nés prématurés : apport des données épidémiologiques. Enfance 2013;2013:49-58.

[8] Lejeune F, Gentaz E. L'enfant prématuré en 2018 : multiplicité des enjeux. Approche Neuropsychol Apprentiss Chez Enfant 2018:17-24.

[9] Bullinger A. Le développement sensori-moteur de l'enfant et ses avatars. Toulouse: Erès; 2015.

[10] Vaivre-Douret L, Papiernik E, Relier JP. Méthode et soins kangourou. Arch Pédiatrie 1996;3:1262-1269.

[11] Pierrat V, Bomy H, Courcel C, Dumur S, Caussette V, Bouckenhove N, et al. Le peau à peau dans la prise en charge des nouveau-nés de faible poids de naissance. J Pédiatrie Puériculture 2004;17:351-7.

[12] Buil A, Fillon-Devys D, Granger A, Roger K, Thomas N, Apter G, et al. Impact de l'installation en Flexion diagonale soutenue sur le maternage tactile spontané lors de la première séance de peau-à-peau en réanimation néonatale. Neuropsychiatr Enfance Adolesc 2017;65:289-98.

[13] Vasseur R, Delion P. Périodes sensibles dans le développement psychomoteur de l'enfant de 0 à 3 ans. Toulouse: Erès; 2010.

[14] Gentaz E. Toucher pour connaître: Psychologie cognitive de la perception tactile manuelle Chapitre 1. Caractéristiques générales de l'organisation anatomo-fonctionnelle de la perception cutanée et haptique. Paris: Presses universitaires de France; 2000.

[15] Diego MA, Field T, Hernandez-Reif M, Deeds O, Ascencio A, Begert G. Preterm infant massage elicits consistent increases in vagal activity and gastric motility that are associated with greater weight gain. Acta Pædiatrica 2007;96:1588-91.

[16] Zerbini A. Comprendre le développement psychologique des prématurés. CQFD 2015.

[17] Sandre D. Soins de développement et prévention des difficultés développementales chez les bébés nés prématurément. Spirale 2016:109-13.

[18] Muller J-B, Castaing V, Denizot S, Caillaux G, Frondas A, Simon L, et al. Le programme NIDCAP (Newborn Individualized Developmental Care and Assessment Program). Principes et théorie. Mot Cérébrale Réadapt Neurol Dév 2014;35:41-3.

[19] Sizun J, Ansquer H, Browne J, Tordjman S, Morin J-F. Developmental care decreases physiologic and behavioral pain expression in preterm neonates. J Pain 2002;3:446-50.

[20] Spittle A, Orton J, Anderson PJ, Boyd R, Doyle LW. Early developmental intervention programmes provided post hospital discharge to prevent motor and cognitive impairment in preterm infants. Cochrane Database Syst Rev 2015. 
[21] Cioni G, Ferrari F, Einspieler C, Paolicelli P, Barbani M, Prechtl H. Comparison between observation of spontaneous movements and neurologic examination in preterm infants. $\mathbf{J}$ Pediatr 1997;5:704-11.

[22] Dehaene S. Fondements cognitifs des apprentissages scolaires. Education, plasticité cérébrale et recyclage neuronal. 2015.

[23] Haute Autorité de Santé. Recommandation de bonne pratique. Troubles du neurodéveloppement. Repérage et orientation des enfants à risque 2020.

[24] Beaud S. L'usage de l'entretien en sciences sociales. Plaidoyer pour l'«entretien ethnographique». Politix 1996;9:226-57.

[25] Pinson G, Sala Pala V. Peut-on vraiment se passer de l'entretien en sociologie de l'action publique? Rev Fr Sci Polit 2007;57:555.

[26] Broqua C. Enjeux des méthodes ethnographiques dans l'étude des sexualités entre hommes1. J Anthropol 2000:129-55.

[27] Becker HS. Les ficelles du métier: comment conduire sa recherche en sciences sociales. Paris: La Découverte; 2013.

[28] Lizé W. Entretiens, directivité et imposition de problématique. Une enquête sur le goût musical. Genèses 2009;76:99.

[29] Durkheim E. Les règles de la méthode sociologique. 16ème édition. Paris: Presses universitaires de France; 1967.

[30] Bourdieu P, Chamboredon J-C, Passeron JC. Le métier de sociologue: préalables epistémologiques. 2. éd. Paris: Mouton; 1973.

[31] Goffman E. Les cadres de l'expérience. Paris: Minuit; 1991.

[32] Broqua C. L'ethnographie comme engagement : enquêter en terrain militant. Genèses 2009;75:109.

[33] Bourdieu P. Systèmes d'enseignement et systèmes de pensée. Fonct Soc Léducation 1967:367-88.

[34] Kaufmann J-C, Singly F de. L'entretien compréhensif. Paris: Armand Colin; 2004.

[35] Murcier N. La réalité de l'égalité entre les sexes à l'épreuve de la garde des jeunes enfants. Mouvements 2007;49:53-62.

[36] Bourdieu P. La domination masculine. Éd. augm. d'une préface. Paris: Ed. du Seuil; 2002.

[37] Becker HS. Les Aspects Concrets de la Thèse 2011.

[38] Beaud S, Weber F. Guide de l'enquête de terrain: produire et analyser des données ethnographiques. Paris: La Découverte; 2017.

[39] Fontaine A. La recherche ethnographique en travail social : l'exemple d'une étude de cas sur le travail de rue. Pensée Plurielle 2012;n 30-31:83.

[40] Pruvost G. La production d'un récit maîtrisé : les effets de la prise en note des entretiens et de la socialisation professionnelle.: Le cas d'une enquête dans la police. Lang Société 2008; 123:73.

[41] Pires A. "Échantillonnage et recherche qualitative : essai théorique et méthodologique." Rech. Qual. Enjeux Épistémologiques Méthodologiques, Montréal: Gaetan Morin Editeur; 1997, p. 113-69.

[42] Bourdieu P, Eicher JC. Capital humain, capital culturel. Ecole Normale Supérieure de St Cloud; 1977.

[43] Fleury C, Ameisen JC. Leçon inaugurale chaire humanité et santé 07/12/2018 n.d.

[44] Weber M, Kalinowski I, Sintomer Y. La domination. Paris: La Découverte; 2015.

[45] Memmi D, Arduin P. L'enquêteur enquêté. De la «connaissance par corps » dans l'entretien sociologique. Genèses 1999;35:131-45.

[46] Bloy G. Échec des messages préventifs et gouvernement des conduites en médecine générale. Sci Soc Santé 2015:41-66. 
[47] Boursier P, Fondation Copernic, editors. Manuel indocile de sciences sociales: pour des savoirs résistants. Paris: La Découverte; 2019.

[48] Juven P-A. Une santé qui compte? les coûts et les tarifs controversés de l'hôpital public. 1re édition. Paris: Puf; 2016.

[49] Morgan AS, Khoshnood B, Diguisto C, Foix L'Helias L, Marchand-Martin L, Kaminski $\mathrm{M}$, et al. Intensity of perinatal care for extremely preterm babies and outcomes at a higher gestational age: evidence from the EPIPAGE-2 cohort study. BMC Pediatr 2020;20:8.

[50] Monier I, Ancel P-Y, Ego A, Guellec I, Jarreau P-H, Kaminski M, et al. Gestational age at diagnosis of early-onset fetal growth restriction and impact on management and survival: a population-based cohort study. BJOG Int J Obstet Gynaecol 2017;124:1899_ 906.

[51] Avenel C. Transitions identitaires chez les entrants en études de médecine. Quest Vives Rech En Éducation 2015.

[52] Bourdieu P. La noblesse d'état: grandes écoles et esprit de corps. Paris: Les Éd. de Minuit; 2002.

[53] Foucault M. Radioscopie n.d.

[54] Laisné C, Heyman I. Problèmes actuels d'hospitalisme en néonatalogie: Deux exemples en Martinique. Coq-Héron 2005;181:68.

[55] Bertrand X. Décret $n^{\circ} 2006-74$ du 24 janvier 2006 relatif aux conditions techniques de fonctionnement auxquelles doivent satisfaire les établissements de santé pour pratiquer les activités de réanimation pédiatrique et de surveillance continue pédiatrique. 2006.

[56] Pierru F. Impératifs gestionnaires et phronesis médicale : esquisse sociologique d'un engagement éthique dans un grand hôpital parisien. Quad Commun Technol Pouvoir 2013:67-82.

[57] Pierru F. Les mandarins à l'assaut de l'usine à soins. Bureaucratisation néolibérale de l'hôpital français et mobilisation de l'élite hospitalo-universitaire. Bur. Néolibérale, Paris: Éditions La Découverte; 2013, p. 203-30.

[58] Juven P-A, Pierru F, Vincent F. La casse du siècle: à propos des réformes de l'hôpital public. 2019.

[59] Georges-Tarragano. Soigner (1')humain. Presses de l'EHESP. Rennes: 2015.

[60] Freidson E. La Profession médicale. Paris: Payot; 1984.

[61] Hugues EC. The making of a physician. General Statement of Ideas and problems. Hum Organ 1956;14:21-5.

[62] Toffel K, Bühlmann F, Tawfik A. L'espace professionnel infirmier : une analyse à partir du cas de la Suisse romande. Rev Fr Sociol 2018;59:219.

[63] Schweyer F-X, Metzger J-L. Entre profession, organisation et marché : le cas des ingénieurs biomédicaux hospitaliers. Rev Fr Aff Soc 2005;1:183.

[64] Schwartz O. L'empirisme irréductible. La fin de l'empirisme? Hobo Sociol. Un -Abri, Paris: Armand Colin; 2014, p. 335-84.

[65] Juven P-A. Produire l'information hospitalière: Du codage des dossiers au gouvernement de l'activité. Rev Anthropol Connaiss 2013;7, 4:815.

[66] Juven P-A, Gaudillière J-P. «En consultation, je regarde l'ordinateur, pas ma patiente »: Entretien avec Anne Gervais. Mouvements 2019; ${ }^{\circ} 98: 23$.

[67] Elger B. Le paternalisme médical mythe ou réalité?: aspects philosophiques et empiriques d'un phénomène persistant. Chêne-Bourg: Editions Médecine \& Hygiène; 2010.

[68] Salbreux R. Contexte de l'évolution des rapports parents-professionnels au cours du dernier demi-siècle. Contraste 2013;37:47. 
[69] Bloch M, Hénaut L, Sardas J, Gand S. La coordination dans le champ sanitaire et médico-social. Enjeux organisationnels et dynamiques professionnelles. Paris: Centre de gestion scientifique de Mines-ParisTech; 2011.

[70] Baudouin J-M, Friedrich J. Théorie de l'action et éducation. De Boeck Supérieur. Louvain-La-Neuve, Belgique: 2001.

[71] Canguilhem G. Le normal et le pathologique. PUF. Paris: 1991.

[72] Lériche R. Introduction générale; De la santé à la Maladie ; La douleur dans les maladies ; Où va la médecine? Encycl. Fr. T VI, 1936. 